\title{
Sulfur Dioxide and Nitrogen Dioxide Meet Growth, Gas Exchange, and Water Relations of Potato Plants
}

\author{
Jane M. Petitte ${ }^{1}$ and Douglas P. Ormrod \\ Department of Horticultural Science, University of Guelph, Guelph, Ont. N1G 2W1 Canada
}

Additional index words. Solarium tuberosum, air pollution, water potential, stomatal conductance, photosynthesis, water loss

\begin{abstract}
The effects of $\mathrm{SO}_{2}$ and $\mathrm{NO}_{2}$, singly and in combination, on the growth and physiology of nontuberizing Solarium tuberosum $\mathrm{L}$. 'Russet Burbank' plants were studied in controlled conditions. Plants were exposed to $0.11 \mu \mathrm{l}$ $\mathrm{SO}_{2}$ and/or $0.11 \mu \mathrm{N} \mathrm{NO}$ /liter for 24 hours a day up to 10 days. Statistically significant effects were observed mainly in the $\mathrm{SO}_{2}+\mathrm{NO}_{2}$ treatments compared with the control plants. Leaf area was reduced from day 2 onward, and root fresh and dry weights were reduced from day 4 onward. Significant reductions in leaf and stem dry weights occurred on day 6. Net $\mathrm{CO}_{2}$ exchange rates were reduced for $\mathrm{SO}_{2}$ exposed compared with control plants beginning on day 3 , while water loss rates were increased with $\mathrm{SO}_{2}+\mathrm{NO}_{2}$ beginning on day 3 . The increases in water loss rate were possibly due to the development of cuticular injury observed as abaxial glazing on the upper and middle canopy leaves. Leaf osmotic potential $(\pi)$ of plants with $\mathrm{SO}_{2}+\mathrm{NO}_{2}$ became more negative within the first 24 hours of the exposure. This reduction was accompanied by an increase in reducing sugar concentration. Xylem water potential was reduced in the mature and expanding leaflets by day 2 of the $\mathrm{SO}_{2}+\mathrm{NO}_{2}$ exposure. The most sensitive aspect of the action of $\mathrm{SO}_{2}+\mathrm{NO}_{2}$ appeared to be the increase in reducing sugars that affected osmotic potential in the leaves. Considering the retardation of root growth, these data suggest that the pollutant gases may have interfered with partitioning of dry matter from the leaves to the roots.
\end{abstract}

Potato plants demonstrate various responses to $\mathrm{SO}_{2}$ and $\mathrm{NO}_{2}$. Sulfur dioxide exposure causes reductions in tuber yield and mean tuber size (Foster et al., 1983), while chronic $\mathrm{NO}_{2} \mathrm{ex}-$ posures throughout the growing season cause reductions in tuber number and fresh weight (Sinn and Pen, 1984). Sulfur dioxide $+\mathrm{NO}_{2}$ alters potato plant water status and interferes with the partitioning of dry matter from the leaves to the roots (Petitte and Ormrod, 1988).

At low doses, air pollutants may alter biochemical or physiological cell processes (e.g., stomatal function in photosynthesis) resulting in lowered plant productivity without visible injury (Heath, 1980). These alterations can be the result of several factors. Exposure of soybean (Amundson and Weinstein, 1981) and garden bean (Ashenden, 1979b) to $\mathrm{SO}_{2}$ and $\mathrm{NO}_{2}$ caused parallel decreases in transpiration and photosynthesis rates due to increased stomatal resistance. However, Sinn and Pen (1984) demonstrated that 'Kennebec' and 'Atlantic' potato plants chronically exposed to levels of $\mathrm{NO}_{2}$ ranging from 0.12 to 0.34 $\mu \mathrm{l} \cdot$ liter $^{-1}$ did not experience significant increases in leaf diffusive resistance. Reinert (1984) stated that the reductions in photosynthesis rates, as a result of plant exposure to some combinations of $\mathrm{SO}_{2}$ and $\mathrm{NO}_{2}$, suggest an increase in respiration. Carlson (1983) believed that where reductions in photosynthesis were not the result of increases in respiration (Black and Unsworth, 1979), the reduction was due to increased internal diffusive resistances. A reduction in photosynthesis also may be caused by the actions of pollutant by-products formed in the leaves. Malhotra and Khan (1984) state that $\mathrm{SO}_{2}$ can affect

\footnotetext{
Received for publication 16 Apr. 1990. This paper is a portion of a thesis submitted by J.M.P. in fulfilling the requirements for a PhD. Financial assistance for this research was provided by an operating grant from the Natural Sciences and Engineering Research Council of Canada. The cost of publishing this paper was defrayed in part by the payment of page charges. Under postal regulations, this paper therefore must be hereby marked advertisement solely to indicate this fact.

'Current address: 620 Shadywood Lane, Raleigh, NC 27603.
}

photosynthesis by influencing the carboxylation reactions and by attacking photosynthetic electron transport and photophosphorylation reactions. Reductions in photosynthesis in the potato affect metabolism of assimilates needed to maximize tuber production and quality (Harris and Pittman, 1923). No work has been reported concerning the effects of $\mathrm{SO}_{2}$ and/or $\mathrm{NO}_{2}$ on photosynthesis or water loss in the potato.

Maintenance of water status in the potato plant is necessary for continued production of photosynthetically active leaf area (Gandar and Tanner, 1976). However, $\psi_{\text {lear }}$ can be reduced by $\mathrm{SO}_{2}$ in combination with $\mathrm{O}_{3}$, as was observed in 'White Cascade' Petunia (Elkiey and Ormrod, 1979). The reduction in $\psi_{\text {leat }}$ may be due to an alteration in $\pi$ as influenced by increasing free sugars in the leaf tissue. Koziol and Jordan (1978) observed in garden bean an increase in free sugars at low $\mathrm{SO}_{2}$ concentrations, which they suggested was important in the repair process when little or no injury was visible. We found no reports elucidating the effects of $\mathrm{SO}_{2}$ and/or $\mathrm{NO}_{2}$ on $\psi_{\text {leaf }}$ and its components.

Air quality data on $\mathrm{SO}_{2}$ and $\mathrm{NO}_{2}$ in the United States (Tingey et al., 1971) and the United Kingdom (Ashenden, 1979a) have indicated that the occurrence of combinations of these gases is possible (Ormrod, 1982). Combinations of $\mathrm{SO}_{2}$ and $\mathrm{NO}_{2}$ can affect growth (Ashenden, 1979a; Tingey et al., 1971) in many plant species, even when the same concentration of either pollutant alone does not cause injury. The concentrations of $\mathrm{SO}_{2}$ and $\mathrm{NO}_{2}$ in our study are based upon the maximum 24-h level for a single gas as presented in the Recommended Air Quality Objectives of Environment Canada (Fisheries and Environment Canada, 1976). The purpose of this experiment was to determine the time course of effects of the single and mixed gases upon shoot and root growth, $\mathrm{CO}_{2}$ exchange, water loss, $\psi_{\text {leat }}$, and reducing sugar content of leaves of the potato plant. Abbreviations: CSTR, continuously stirred tank reactor; PLA, planar leaf area;
$\pi$, osmotic potential; $\psi_{\text {leaf }}$ leaf water potential. 


\section{Materials and Methods}

Propagation of experimental plants. Stem cuttings were taken from plants grown from 12-month-old 'Russest Burbank' tubers and rooted as described by Petitte and Ormrod (1988). Rooted stem cuttings were transplanted into a mixture of 1 Premix BX (Premier Brands, Stamford, Corm.) :1 Fox sandy loam (v/v). Ten days later, plants were taken at random for experimentation.

Experimental design. There were two studies. Plant growth, photosynthesis, water loss, stomatal conductance, and water potential were measured in the first. Reducing sugars were measured in the second. Both studies were repeated three times over succeeding months with extra replications conducted for water potential measurement in the first study. The design for each study was a randomized complete block with the repetitions of the study as blocks. Data for each response variable were analyzed separately for each time of measurement. The growth variables were transformed to their natural logarithms before analysis of covariance with PLA (Ormrod et al., 1983) before treatment as the covariate. Geometric least mean squares were obtained for the growth variables by computing the antilog of means of transformed values (Snedecor and Cochran, 1967). Data for all other response variables were subjected to analysis of variance (ANOVA). The onset of treatment effects with increasing duration of treatment was detected on the basis of significance of contrasts between treated and control plants measured at the same hourly time. When the contrast was significant, the onset of treatment effects was deemed to have taken place.

Exposure conditions. In each replicate of the first study, 10 plants were placed into each of four CSTR chambers (Le SueurBrymer, 1982). In each replicate of the second study, 11 plants were placed into each CSTR. The exposure conditions inside the CSTR for both studies were: day/night at 25/18C $\pm 1 \mathrm{C}$; relative humidity, $79 \% \pm 5 \%$; photosynthetically active radiation (PAR), $325 \pm 30 \mu \mathrm{mol} \cdot \mathrm{m}^{-2} \cdot \mathrm{s}^{-1}$ with a $16 \mathrm{~h}$-day ${ }^{-1}$ photoperiod (0600-2200 HR) supplied by a 400-W high-pressure sodium lamp and a 400-W metal halide lamp. A LI-COR LI185 (LI-COR, Lincoln, Neb.) meter was used to measure PAR at mid-canopy level. Air temperature and relative humidity inside each CSTR chamber were monitored using a Taylor hygrometer (Model 5522, Taylor Instruments/Sybrong Corp., Arden, N. C.) situated at midcanopy level. Air flow rate through each CSTR chamber was 130 liters.min ${ }^{-1}$, measured periodically with an Alnor thermoanemometer (Type 8500, Alnor Instrument Co., Skokie, Ill.). The plants were irrigated daily with full strength complete nutrient solution (Hoagland and Agnon, 1950). Following $\approx 18 \mathrm{~h}$ of acclimation to the CSTR conditions and beginning at $\approx 0900 \mathrm{HR}$ on Day 0 , the plants were exposed for 24 $\mathrm{h} \cdot \mathrm{day}^{-\mathrm{T}}$ to one of the following treatments: 1) filtered air (no detectable $\mathrm{SO}_{2}$ or $\mathrm{NO}_{2}$ ); 2) $0.11 \mathrm{SO}_{2}$; 3) $0.11 \mathrm{NO}_{2}$; or 4) 0.11 $\mathrm{SO}_{2}$ plus $0.11 \mu \mathrm{NO}_{2} /$ liter for a maximum of 10 days. Sulphur dioxide and $\mathrm{NO}_{2}$ were supplied from gas cylinders; a Beckman Model $953 \mathrm{SO}_{2}$ analyzer, and a Beckman Model 952 $\mathrm{A} \mathrm{NO}_{2}$ analyzer comprised the monitoring system (Beckman Instruments, Fullerton, Calif.). Analyzers were calibrated with $\mathrm{SO}_{2}$ and NO calibration tank gases supplied by the Speciality Gas Division of Liquid Carbonic Ltd. The NO was used in a Thermoelectron Series 101 calibrator (Thermo Electron, Hopkinton, Mass.) to calibrate for $\mathrm{NO}_{2}$.

Net carbon dioxide exchange rates and water loss rates. Inlet and outlet $\mathrm{CO}_{2}$ concentrations for each CSTR chamber were continuously monitored by a Beckman Model 865 infrared analyzer to permit calculation of net $\mathrm{CO}_{2}$ exchange rates. A General Eastern 1100 dew point hygrometer (General Eastern Instru- ments, Watertown, Mass.) continuously monitored inlet and outlet dew point temperatures of each chamber to permit calculation of water loss rates.

Leaf areas for determining net $\mathrm{CO}_{2}$ exchange rate and water loss rates within each CSTR chamber at 0300, 1100, and 1900 HR were estimated in the following manner. Before placement into the CSTR chamber, a covariate, PLA (Ormrod et al., 1983), was determined for each plant. Several extra plants were harvested to determine the linear relationship between PLA and leaf area. Leaf area was measured using a LI-COR LI-3100 leaf area meter. By substituting the PLA for each plant into the linear regression equation calculated from extra plants, an approximation of the initial leaf area for each experimental plant could be calculated.

Throughout the 10-day exposure, following measurements for stomatal conductance and water potential, two plants from each treatment were harvested (one at $\approx 1100 \mathrm{HR}$ and the second at $1700 \mathrm{HR}$ ) every 2 days to measure leaf area and leaf, stem, and root fresh and dry weights. After each plant was harvested, the difference between the actual measured leaf area and the estimated initial leaf area was calculated. The changes in leaf area for plants in each CSTR chamber were regressed over the 10day exposure. The regression equation for each treatment was used to calculate the approximate photosynthetically active leaf area remaining in the CSTR chamber at 0300, 1100, and 1900 HR for each day of the exposure. The approximate photosynthetically active leaf area was then used to calculate net $\mathrm{CO}_{2}$ exchange water loss rates for each time (Petitte, 1986).

Pots containing soil at field capacity were placed into each CSTR chamber to determine net $\mathrm{CO}_{2}$ exchange and water loss not due to the plant. These values were incorporated into the daily $\mathrm{CO}_{2}$ exchange rates and water loss rates for each treatment in each block.

Stomatal conductance. Stomatal conductance was measured using a LI-COR model LI-700 transient porometer. Conductance measurements were made on days $0,1,2,4,6,8$, and 10 at $\approx 4$ and $10 \mathrm{~h}$ after the beginning of the 16 - $\mathrm{h}$ photoperiod. The terminal leaflets of the fifth, sixth, or seventh leaf from the soil line (mature, lower canopy) and the first or second newly unfurled leaf from the shoot apex (expanding, upper canopy) were measured. The CSTR chamber door was opened wide enough to move the cuvette of the porometer through the opening and clamp it onto a terminal leaflet. To avoid a buildup of $\mathrm{CO}_{2}$ in the CSTR chambers, the person making the measurements wore a mask connected to an external vacuum pump over the nose and mouth. A single but different plant was used for each day's morning and afternoon readings.

Water potential. Water potential measurements were taken on the same terminal leaflets of mature and expanding leaves as used for the stomatal conductance readings every 2 days up to and including day 10 beginning on day 2 . Preliminary statistical analysis indicated significant changes on the morning of day 2 in water potential measurements between the control and the plants that had been exposed to the mixture. Additional replications were conducted to permit measurements to be made at $6 \mathrm{~h}$ (afternoon of day O), $24 \mathrm{~h}$ (morning of day 1), $30 \mathrm{~h}$ (afternoon of day 1),48 h (morning of day 2), and $52 \mathrm{~h}$ (afternoon of day 2) after the initiation of the exposure.

For measurement of $\psi_{\text {leaf }}$ the leaflet was loosely wrapped with a moist paper tissue. Then the leaflet with $\approx 1 \mathrm{~cm}$ of intact petiole was excised from the leaf with a sharp razor blade. The $\psi_{\text {leaf }}$ was measured in terms of xylem water potential using a pressure bomb that was lined with moist paper towels. 
Table 1. Growth of nontuberizing 'Russet Burbank' potato plants exposed to filtered air or SO, and NO, for 10 days.

\begin{tabular}{|c|c|c|c|c|c|c|c|c|c|}
\hline \multirow[b]{2}{*}{ Characteristics } & \multicolumn{2}{|c|}{$\begin{array}{c}\begin{array}{c}\text { Pollutant } \\
\text { concn } \\
\left(\mu l \cdot \text { liter }^{-1}\right)\end{array} \\
\end{array}$} & \multicolumn{5}{|c|}{ Days of exposure } & \multirow[b]{2}{*}{$r^{2}$} & \multirow[b]{2}{*}{ Response equation } \\
\hline & $\mathrm{SO}_{2}$ & $\mathrm{NO}_{2}$ & 2 & 4 & 6 & 8 & 10 & & \\
\hline $\begin{array}{l}\text { Leaf area } \\
\qquad\left(\mathrm{cm}^{2} / \text { plant }\right)\end{array}$ & $\begin{array}{l}0.0 \\
0.11\end{array}$ & $\begin{array}{l}0.0 \\
0.11\end{array}$ & $\begin{array}{l}908 \\
788^{*}\end{array}$ & $\begin{array}{l}1130 \\
897^{*}\end{array}$ & $\begin{array}{l}1560 \\
1070^{*}\end{array}$ & $\begin{array}{l}1930 \\
1090^{*}\end{array}$ & $\begin{array}{l}2370 \\
1210^{*}\end{array}$ & $\begin{array}{l}0.99 \\
0.84\end{array}$ & $\begin{array}{l}y^{z}=624+86 x^{y}+8 x^{2} \\
y=643+55 x\end{array}$ \\
\hline $\begin{array}{l}\text { Leaf dry wt } \\
\text { (g/plant) }\end{array}$ & $\begin{array}{l}0.0 \\
0.11\end{array}$ & $\begin{array}{l}0.0 \\
0.11\end{array}$ & $\begin{array}{l}2.17 \\
2.06\end{array}$ & $\begin{array}{l}2.84 \\
2.62\end{array}$ & $\begin{array}{l}4.18 \\
3.44^{*}\end{array}$ & $\begin{array}{l}5.26 \\
3.53^{*}\end{array}$ & $\begin{array}{l}6.83 \\
4.60\end{array}$ & $\begin{array}{l}0.95 \\
0.90\end{array}$ & $\begin{array}{l}y=1.43+0.20 x+0.032 x^{2} \\
y=1.28+0.3 x\end{array}$ \\
\hline $\begin{array}{l}\text { Stem dry wt } \\
. \text { (g/plant) }\end{array}$ & $\begin{array}{l}0.0 \\
0.11\end{array}$ & $\begin{array}{l}0.0 \\
0.11\end{array}$ & $\begin{array}{l}0.760 \\
0.724\end{array}$ & $\begin{array}{l}1.20 \\
1.15\end{array}$ & $\begin{array}{l}1.92 \\
1.50^{*}\end{array}$ & $\begin{array}{l}2.57 \\
1.77^{*}\end{array}$ & $\begin{array}{l}3.52 \\
2.26^{*}\end{array}$ & $\begin{array}{l}0.95 \\
0.96\end{array}$ & $\begin{array}{l}y=0.90-0.036 x+0.028 x^{2} \\
y=0.28+0.19 x\end{array}$ \\
\hline $\begin{array}{l}\text { Root dry wt } \\
\text { (g/plant) }\end{array}$ & $\begin{array}{l}0.0 \\
0.11 \\
\end{array}$ & $\begin{array}{l}0.0 \\
0.11 \\
\end{array}$ & $\begin{array}{l}0.658 \\
0.558 \\
\end{array}$ & $\begin{array}{l}0.818 \\
0.629^{*}\end{array}$ & $\begin{array}{l}0.945 \\
0.770^{*} \\
\end{array}$ & $\begin{array}{l}1.24 \\
0.728^{*} \\
\end{array}$ & $\begin{array}{l}1.57 \\
0.886^{*}\end{array}$ & $\begin{array}{l}0.92 \\
0.68\end{array}$ & $\begin{array}{l}y=0.70+0.034 x+0.01 x^{2} \\
y=0.46+0.039 x\end{array}$ \\
\hline
\end{tabular}

zy: Response variable.

y: Days.

*Significantly less than the control $(0.0)$ in the same column for the same criterion $(P \leq 0.05)$.

Table2. Net $\mathrm{CO}_{2}$ exchange rates $\left(\mathrm{mg} \cdot \mathrm{m}^{-2} \cdot \mathrm{s}^{-1}\right)$ of nontuberizing 'Russet Burbank' potato plants exposed to $\mathrm{SO}_{2}$ and/or $\mathrm{NO}_{2}$ during 10 days.

\begin{tabular}{|c|c|c|c|c|c|c|}
\hline \multirow[b]{2}{*}{ Day } & \multirow[b]{2}{*}{$\begin{array}{c}\operatorname{Time}^{z} \\
(\mathrm{HR})\end{array}$} & \multicolumn{5}{|c|}{ Pollutant concn $\left(\mu \mathrm{l} \cdot\right.$ liter $\left.^{-1}\right)$} \\
\hline & & $\begin{array}{l}\mathrm{SO}_{2} \\
\mathrm{NO}_{2} \\
\end{array}$ & $\begin{array}{l}0.0 \\
0.0\end{array}$ & $\begin{array}{l}0.11 \\
0.0 \\
\end{array}$ & $\begin{array}{l}0.0 \\
0.11 \\
\end{array}$ & $\begin{array}{l}0.11 \\
0.11\end{array}$ \\
\hline 0 & 1900 & & 0.129 & $0.106^{*}$ & 0.128 & 0.124 \\
\hline 1 & $\begin{array}{l}0300 \\
1100 \\
1900\end{array}$ & & $\begin{array}{r}-0.006 \\
0.158 \\
0.128\end{array}$ & $\begin{array}{r}-0.000 \\
0.126 \\
0.112\end{array}$ & $\begin{array}{r}-0.008 \\
0.151 \\
0.128\end{array}$ & $\begin{array}{r}-0.008 \\
0.138 \\
0.125\end{array}$ \\
\hline 2 & $\begin{array}{l}0300 \\
1100 \\
1900\end{array}$ & & $\begin{array}{r}-0.005 \\
0.148 \\
0.133\end{array}$ & $\begin{array}{r}-0.002 \\
0.127 \\
0.117\end{array}$ & $\begin{array}{r}-0.004 \\
0.143 \\
0.135\end{array}$ & $\begin{array}{r}-0.009 \\
0.122 \\
0.128\end{array}$ \\
\hline 3 & $\begin{array}{l}0300 \\
1100 \\
1900\end{array}$ & & $\begin{array}{r}-0.012 \\
0.148 \\
0.129\end{array}$ & $\begin{array}{c}-0.007 \\
0.120^{*} \\
0.110\end{array}$ & $\begin{array}{r}-0.009 \\
0.149 \\
0.120\end{array}$ & $\begin{array}{r}-0.015 \\
0.125 \\
0.116\end{array}$ \\
\hline 4 & $\begin{array}{l}0300 \\
1100 \\
1900\end{array}$ & & $\begin{array}{r}-0.010 \\
0.154 \\
0.140\end{array}$ & $\begin{array}{r}-0.008 \\
0.127 \\
0.118\end{array}$ & $\begin{array}{r}-0.010 \\
0.152 \\
0.135\end{array}$ & $\begin{array}{r}-0.014 \\
0.126 \\
0.123\end{array}$ \\
\hline 5 & $\begin{array}{l}0300 \\
1100 \\
1900\end{array}$ & & $\begin{array}{r}-0.015 \\
0.157 \\
0.132\end{array}$ & $\begin{array}{c}-0.012 \\
0.123^{*} \\
0.116\end{array}$ & $\begin{array}{r}-0.012 \\
0.153 \\
0.139\end{array}$ & $\begin{array}{c}-0.014 \\
0.128^{*} \\
0.113\end{array}$ \\
\hline 6 & $\begin{array}{l}0300 \\
1100 \\
1900\end{array}$ & & $\begin{array}{r}-0.014 \\
0.161 \\
0.143\end{array}$ & $\begin{array}{c}-0.013 \\
0.124^{*} \\
0.117\end{array}$ & $\begin{array}{r}-0.014 \\
0.162 \\
0.150\end{array}$ & $\begin{array}{r}-0.017 \\
0.130 \\
0.115\end{array}$ \\
\hline 7 & $\begin{array}{l}0300 \\
1100 \\
1900\end{array}$ & & $\begin{array}{r}-0.021 \\
0.162 \\
0.145\end{array}$ & $\begin{array}{c}-0.014 \\
0.119^{*} \\
0.114\end{array}$ & $\begin{array}{r}-0.018 \\
0.166 \\
0.145\end{array}$ & $\begin{array}{r}-0.020 \\
0.133 \\
0.118\end{array}$ \\
\hline 8 & $\begin{array}{l}0300 \\
1100 \\
1900\end{array}$ & & $\begin{array}{r}-0.017 \\
0.181 \\
0.146\end{array}$ & $\begin{array}{r}-0.013 \\
0.116^{* *} \\
0.108^{* *}\end{array}$ & $\begin{array}{r}-0.019 \\
0.165 \\
0.151\end{array}$ & $\begin{array}{c}-0.016 \\
0.123^{* *} \\
0.121^{*}\end{array}$ \\
\hline 9 & $\begin{array}{l}0300 \\
1100 \\
1900\end{array}$ & & $\begin{array}{r}-0.020 \\
0.149 \\
0.140\end{array}$ & $\begin{array}{r}-0.013 \\
0.111 \\
0.108\end{array}$ & $\begin{array}{r}-0.026 \\
0.190 \\
0.148\end{array}$ & $\begin{array}{c}-0.025 \\
0.112 \\
0.106^{*}\end{array}$ \\
\hline 10 & $\begin{array}{l}0300 \\
1.100\end{array}$ & . & $\begin{array}{r}-0.020 \\
0.177\end{array}$ & $\begin{array}{c}-0.016 \\
0.109^{*}\end{array}$ & $\begin{array}{c}-0.028^{*} \\
0.183\end{array}$ & $\begin{array}{r}-0.022 \\
0.127\end{array}$ \\
\hline
\end{tabular}

${ }^{2}$ Photoperiod $=0600$ to $2200 \mathrm{HR}$.

*,** Significantly different from the control in the same row at $\mathrm{P} \leq$ 0.05 or 0.01 , respectively.

After the leaflet was removed from the pressure bomb and the paper tissue was removed, the leaflet was sealed in a plastic
Table 3. Water loss rates $\left(\mathrm{mg} \cdot \mathrm{m}^{-2} \cdot \mathrm{s}^{-1}\right)$ of nontuberizing 'Russet Burbank' potato plants exposed to $\mathrm{SO}_{2}$ and/or $\mathrm{NO}_{2}$ during 10 days.

\begin{tabular}{|c|c|c|c|c|c|c|}
\hline \multirow[b]{2}{*}{ Day } & \multirow[b]{2}{*}{$\begin{array}{c}\operatorname{Time}^{\mathrm{z}} \\
\text { (HR) }\end{array}$} & \multicolumn{5}{|c|}{ Pollutant concn $\left(\mu \mathrm{l} \cdot\right.$ liter $\left.^{-1}\right)$} \\
\hline & & $\begin{array}{l}\mathrm{SO}_{2} \\
\mathrm{NO}_{2} \\
\end{array}$ & $\begin{array}{l}0.0 \\
0.0 \\
\end{array}$ & $\begin{array}{l}0.11 \\
0.0 \\
\end{array}$ & $\begin{array}{l}0.0 \\
0.11 \\
\end{array}$ & $\begin{array}{l}0.11 \\
0.11 \\
\end{array}$ \\
\hline 0 & 1900 & & 15.6 & 14.6 & 15.2 & 16.6 \\
\hline 1 & $\begin{array}{l}0300 \\
1100 \\
1900\end{array}$ & & $\begin{array}{r}4.5 \\
15.0 \\
16.3\end{array}$ & $\begin{array}{r}3.8 \\
14.4 \\
15.7\end{array}$ & $\begin{array}{r}4.6 \\
15.2 \\
15.2\end{array}$ & $\begin{array}{r}4.7 \\
15.1 \\
16.6\end{array}$ \\
\hline 2 & $\begin{array}{l}0300 \\
1100 \\
1900\end{array}$ & & $\begin{array}{r}4.1 \\
17.2 \\
16.6\end{array}$ & $\begin{array}{r}4.4 \\
15.9 \\
16.1\end{array}$ & $\begin{array}{r}4.1 \\
13.2 \\
14.0\end{array}$ & $\begin{array}{r}4.6 \\
17.4 \\
19.4\end{array}$ \\
\hline 3 & $\begin{array}{l}0300 \\
1100 \\
1900\end{array}$ & & $\begin{array}{r}3.7 \\
16.8 \\
14.8\end{array}$ & $\begin{array}{r}3.4 \\
16.3 \\
14.8\end{array}$ & $\begin{array}{r}7.2 \\
14.3 \\
16.5\end{array}$ & $\begin{array}{c}5.2 \\
17.8 \\
25.0^{*}\end{array}$ \\
\hline 4 & $\begin{array}{l}0300 \\
1100 \\
1900\end{array}$ & & $\begin{array}{r}3.4 \\
15.3 \\
14.8\end{array}$ & $\begin{array}{r}3.9 \\
13.6 \\
15.9\end{array}$ & $\begin{array}{c}4.4 \\
10.5 \\
13.8\end{array}$ & $\begin{array}{l}5.1^{*} \\
18.5 \\
23.8^{* *}\end{array}$ \\
\hline 5 & $\begin{array}{l}0300 \\
1100 \\
1900\end{array}$ & & $\begin{array}{r}6.3 \\
13.4 \\
14.8\end{array}$ & $\begin{array}{r}7.6 \\
14.7 \\
14.3\end{array}$ & $\begin{array}{r}7.4 \\
12.6 \\
13.4\end{array}$ & $\begin{array}{c}8.2 \\
23.5^{* *} \\
27.5^{*}\end{array}$ \\
\hline 6 & $\begin{array}{l}0300 \\
1100 \\
1900\end{array}$ & & $\begin{array}{r}4.8 \\
16.1 \\
19.6\end{array}$ & $\begin{array}{r}5.4 \\
18.2 \\
24.1\end{array}$ & $\begin{array}{r}7.2 \\
18.6 \\
18.8\end{array}$ & $\begin{array}{c}7.4 \\
25.8^{* *} \\
31.1^{*}\end{array}$ \\
\hline 7 & $\begin{array}{l}0300 \\
1100 \\
1900\end{array}$ & & $\begin{array}{r}5.2 \\
17.4 \\
19.0\end{array}$ & $\begin{array}{r}6.1 \\
20.2 \\
19.6\end{array}$ & $\begin{array}{r}9.1 \\
15.1 \\
16.0\end{array}$ & $\begin{array}{c}8.4 \\
29.8^{* *} \\
29.5^{* *}\end{array}$ \\
\hline 8 & $\begin{array}{l}0300 \\
1100 \\
1900\end{array}$ & & $\begin{array}{r}5.0 \\
23.3 \\
22.1\end{array}$ & $\begin{array}{r}5.2 \\
23.2 \\
32.4\end{array}$ & $\begin{array}{r}9.3 \\
21.1 \\
33.7\end{array}$ & $\begin{array}{l}8.5 \\
37.6^{* *} \\
39.5\end{array}$ \\
\hline 9 & $\begin{array}{l}0300 \\
1100 \\
1900\end{array}$ & & $\begin{array}{r}6.4 \\
28.8 \\
26.7\end{array}$ & $\begin{array}{r}5.4 \\
32.2 \\
27.3\end{array}$ & $\begin{array}{l}15.2^{*} \\
24.9 \\
25.8\end{array}$ & $\begin{array}{r}6.1 \\
36.5 \\
43.3\end{array}$ \\
\hline 10 & 0300 & & 8.7 & 8.6 & 10.6 & 12.0 \\
\hline
\end{tabular}

${ }^{2}$ Photoperiod $=0600-2200 \mathrm{HR}$.

*** Significantly different from the control in the same row at $P \leq$ 0.05 or 0.01 , respectively.

bag and frozen until IT was determined with a Wescor Model HR-33T microvoltmeter (Wescor, Logan, Utah) operating in a dew point hygrometer mode. For this determination two 5-mm- 
Table 4. Water, osmotic, and pressure potentials $(\mathrm{kPa})$ in expanding and mature leaflets of nontuberizing 'Russet Burbank' plants exposed to $\mathrm{SO}_{2}$ and $\mathrm{NO}_{2}$ during 10 days.

\begin{tabular}{|c|c|c|c|c|c|c|c|c|c|c|}
\hline \multirow[b]{3}{*}{ Day } & \multirow[b]{3}{*}{$\begin{array}{c}\text { Potential } \\
(\mathrm{kPa})\end{array}$} & & \multicolumn{4}{|c|}{ Morning leaflets } & \multicolumn{4}{|c|}{ Afternoon leaflets } \\
\hline & & & \multicolumn{2}{|c|}{ Expanding } & \multicolumn{3}{|c|}{$\begin{array}{l}\text { Mature } \\
\text { Pollutant concn }\left(\mu l \cdot \text { liter }^{-1}\right)\end{array}$} & Expanding & \multicolumn{2}{|c|}{ Mature } \\
\hline & & $\begin{array}{l}\mathrm{SO}_{2} \\
\mathrm{NO}_{2} \\
\end{array}$ & $\begin{array}{l}0.0 \\
0.0 \\
\end{array}$ & $\begin{array}{l}0.11 \\
0.11 \\
\end{array}$ & $\begin{array}{l}0.0 \\
0.0 \\
\end{array}$ & $\begin{array}{l}0.11 \\
0.11 \\
\end{array}$ & $\begin{array}{l}0.0 \\
0.0 \\
\end{array}$ & $\begin{array}{l}0.11 \\
0.11 \\
\end{array}$ & $\begin{array}{l}0.0 \\
0.0 \\
\end{array}$ & $\begin{array}{l}0.11 \\
0.11 \\
\end{array}$ \\
\hline 0 & $\begin{array}{l}\Psi_{\text {leaf }} \\
\pi \\
\mathrm{P}^{\mathrm{z}}\end{array}$ & & & & & & $\begin{array}{r}-295 \\
-709 \\
399\end{array}$ & $\begin{array}{r}-393 \\
-689 \\
303\end{array}$ & $\begin{array}{r}-310 \\
-780 \\
482\end{array}$ & $\begin{array}{r}-360 \\
-753 \\
359^{*}\end{array}$ \\
\hline 1 & $\begin{array}{l}\psi_{\text {leaf }} \\
\mathbf{T} \\
\mathbf{P}\end{array}$ & & $\begin{array}{r}-315 \\
-693 \\
377\end{array}$ & $\begin{array}{c}-342 \\
-838^{* *} \\
526\end{array}$ & $\begin{array}{r}-288 \\
-655 \\
377\end{array}$ & $\begin{array}{c}-335 \\
-769^{* *} \\
443\end{array}$ & $\begin{array}{r}-320 \\
-680 \\
360\end{array}$ & $\begin{array}{c}-388 \\
-722^{* *} \\
385\end{array}$ & $\begin{array}{r}-265 \\
-628 \\
363\end{array}$ & $\begin{array}{c}-348^{*} \\
-757^{* *} \\
409\end{array}$ \\
\hline 2 & $\begin{array}{l}\psi_{\text {leaf }} \\
\mathbf{T} \\
\mathbf{P}\end{array}$ & & $\begin{array}{r}-252 \\
-664 \\
427\end{array}$ & $\begin{array}{c}-352^{* *} \\
-772^{* *} \\
395\end{array}$ & $\begin{array}{r}-212 \\
-628 \\
438\end{array}$ & $\begin{array}{c}-264 \\
-792^{*} \\
504\end{array}$ & $\begin{array}{r}-272 \\
-655 \\
383\end{array}$ & $\begin{array}{c}-412^{* *} \\
-788^{*} \\
376\end{array}$ & $\begin{array}{r}-242 \\
-668 \\
426\end{array}$ & $\begin{array}{c}-320 \\
-814^{* *} \\
494\end{array}$ \\
\hline 4 & $\begin{array}{l}\psi_{\text {leaf }} \\
\pi \\
\mathrm{P}\end{array}$ & & $\begin{array}{r}-285 \\
-718 \\
466\end{array}$ & $\begin{array}{r}-373^{*} \\
-803 \\
414\end{array}$ & $\begin{array}{r}-233 \\
-659 \\
442\end{array}$ & $\begin{array}{c}-317 \\
-826^{* *} \\
521\end{array}$ & $\begin{array}{r}-267 \\
-725 \\
457\end{array}$ & $\begin{array}{c}-392^{*} \\
-826 \\
433\end{array}$ & $\begin{array}{r}-197 \\
-647 \\
449\end{array}$ & $\begin{array}{c}-375^{* *} \\
-923^{* *} \\
548^{-}\end{array}$ \\
\hline 6 & $\begin{array}{l}\Psi_{\text {leaf }} \\
\pi \\
P\end{array}$ & & $\begin{array}{r}-310 \\
-680 \\
386\end{array}$ & $\begin{array}{c}-405^{*} \\
-767^{* *} \\
365\end{array}$ & $\begin{array}{r}-255 \\
-588 \\
340\end{array}$ & $\begin{array}{c}-313 \\
-814^{* *} \\
478^{*}\end{array}$ & $\begin{array}{r}-280 \\
-737 \\
457\end{array}$ & $\begin{array}{c}-433^{* *} \\
-827^{* *} \\
394\end{array}$ & $\begin{array}{r}-207 \\
-628 \\
421\end{array}$ & $\begin{array}{c}-490^{* *} \\
-857^{* *} \\
367\end{array}$ \\
\hline 8 & $\begin{array}{l}\psi_{\text {leaf }} \\
\pi \\
P\end{array}$ & & $\begin{array}{r}-282 \\
-750 \\
475\end{array}$ & $\begin{array}{l}-286 \\
-848^{* *} \\
533\end{array}$ & $\begin{array}{r}-198 \\
-667 \\
469\end{array}$ & $\begin{array}{c}-296^{*} \\
-887^{* *} \\
584\end{array}$ & $\begin{array}{r}-290 \\
-792 \\
502\end{array}$ & $\begin{array}{c}-447^{* *} \\
-943^{* *} \\
495\end{array}$ & $\begin{array}{r}-217 \\
-629 \\
411\end{array}$ & $\begin{array}{c}-612^{* *} \\
-987^{* *} \\
374\end{array}$ \\
\hline 10 & $\begin{array}{l}\psi_{\text {leaf }} \\
\pi \\
\mathrm{P} \\
\end{array}$ & & $\begin{array}{r}-336 \\
-789 \\
447 \\
\end{array}$ & $\begin{array}{r}-536^{*} \\
-880 \\
397\end{array}$ & $\begin{array}{r}-230 \\
-660 \\
437 \\
\end{array}$ & $\begin{array}{c}-430^{*} \\
-894^{* *} \\
557\end{array}$ & $\begin{array}{r}-288 \\
-770 \\
465 \\
\end{array}$ & $\begin{array}{c}-424^{* *} \\
-961^{* *} \\
534\end{array}$ & $\begin{array}{r}-284 \\
-622 \\
347 \\
\end{array}$ & $\begin{array}{c}-428^{*} \\
-911^{* *} \\
541\end{array}$ \\
\hline
\end{tabular}

${ }^{2} \mathbf{P}=$ pressure potential.

${ }_{*, * *}$ Significantly different from the control during the same time period at $P \leq 0.05$ or 0.01 , respectively.

diameter filter paper disks were quickly placed inside the plastic bag beside the frozen leaflet. The bag was resealed and the leaflet was then allowed to thaw. A smooth rounded object was moved firmly over the leaflet in the bag to break up the tissue and allow the liquid to saturate the paper disks. Each disk was quickly removed with clean forceps from the bag and placed into a Wescor C-52 sample chamber. Following a 15-min equilibration, a reading was taken $30 \mathrm{sec}$ after a 10 -sec cooling current (Prange and Ormrod, 1983). The chamber was cleaned with deionized water after each measurement and then dried. The $\pi$ values of two sap samples from each leaflet sample were measured and their mean was used in the ANOVA. Salt solutions of known concentrations were used to calibrate each sample chamber before each replication of the experiment.

Leaflet pressure potential $(\mathrm{P})$ was calculated according to the equation: $\psi_{\text {leaf }}=\pi+\mathrm{m}+\mathrm{P}$, assuming the matric potential (m) to be negligible (Beringer et al., 1983, Wiebe and Al-Saadi, 1976).

Reducing sugars. The second study was conducted to observe reducing sugar concentration during the morning and afternoon in one of the first primary leaflets of mature and expanding leaves during the first 5 days of exposure. Samples of equal area were removed with a cork borer from each lateral half of the leaflet and used for dry weight determination (W) and sugar analyses (S). Total area for the $\mathrm{W}$ or S sample was $77 \mathrm{~mm}^{2}$. The $\mathrm{W}$ samples were dried at $70 \mathrm{C}$ for 5 days and weighed. The $\mathrm{S}$ samples were wrapped in aluminum foil, placed in a plastic bag, and frozen until the time of extraction.

A modification of the technique of Madore (1984) was used to extract the reducing sugars from the leaf disks. The S samples were placed in a test tube with $2 \mathrm{ml}$ of $80 \%$ ethanol. The test tube was placed in boiling water for $\approx 45 \mathrm{sec}$ and then removed. After decanting the liquid from the tissue into a test tube, more ethanol was added, heated, and decanted. The extraction process was repeated two additional times. Preliminary analyses demonstrated that $\approx 95 \%$ of the reducing sugars were removed from the leaf tissue with four extractions. The test tube containing the decanted ethanol extract was covered with two layers of parafilm and placed in a freezer until assayed.

The anthrone assay of Pesez and Bartos (1974) was used for the measurement of reducing sugars in the ethanol extracts. Anthrone $(0.20 \mathrm{~g})$ was dissolved in a cold 5:2 (v/v) mixture of concentrated sulphuric acid and distilled water. Five milliliters of the anthrone reagent was chilled for $5 \mathrm{~min}$ on ice in a test tube. The cold ethanol extract was diluted to $10 \mathrm{ml}$ with cold $80 \%$ ethanol and thoroughly mixed. One milliliter of the extract was layered onto the anthrone reagent and chilled on ice for 5 min. After mixing the extract and the anthrone, the mixture was heated at $100 \mathrm{C}$ in a shaking water bath for 10 rein, cooled for $5 \mathrm{~min}$ in an ice water bath, and the absorbance read at $620 \mathrm{~nm}$ on a Beckman DU-8 spectrophotometer (Beckman Instruments). The standard curve was based on glucose dissolved in $80 \%$ ethanol. Reducing sugar concentration in the first primary leaflets was expressed as milliequivalents of glucose per square millimeter of leaf area.

Precision was defined as the amount of variation within and between assays (Rodbard, 1971). The within- and between-assay $\mathrm{cv}$ were calculated using the ethanol-extracted reducing sugar samples from the control plants. Sensitivity was defined as the amount of glucose that would give an optical reading significantly different from zero, which was calculated as the optical density at zero dose plus twice the SD of the optical density at 
Table 5. Reducing sugars (millequivalent glucose per square millimeter of leaf area) in expanding and mature first primary leaflets of nontuberizing 'Russet Burbank' plants during the first 5 days of a 10-day exuosure to $\mathrm{SO}_{2}$ and/or $\mathrm{NO}_{2}$.

\begin{tabular}{|c|c|c|c|c|c|c|c|}
\hline \multirow[b]{2}{*}{ Day } & \multirow[b]{2}{*}{ Leaf age } & \multirow[b]{2}{*}{ Time $^{x}$} & \multicolumn{5}{|c|}{ Pollutant concn $\left(\mu \mathrm{l} \cdot\right.$ liter $\left.^{-1}\right)$} \\
\hline & & & $\begin{array}{l}\mathrm{SO}_{2} \\
\mathrm{NO}_{2} \\
\end{array}$ & $\begin{array}{l}0.0 \\
0.0 \\
\end{array}$ & $\begin{array}{l}0.11 \\
0.0 \\
\end{array}$ & $\begin{array}{l}0.0 \\
0.11 \\
\end{array}$ & $\begin{array}{l}0.11 \\
0.11 \\
\end{array}$ \\
\hline 0 & $\begin{array}{l}\text { Expanding } \\
\text { Mature }\end{array}$ & $\begin{array}{l}\mathrm{A} \\
\mathrm{A}\end{array}$ & & $\begin{array}{l}1.93 \\
1.26\end{array}$ & $\begin{array}{l}1.41 \\
1.13\end{array}$ & $\begin{array}{l}1.69 \\
1.00\end{array}$ & $\begin{array}{l}2.39 \\
0.90\end{array}$ \\
\hline \multirow[t]{2}{*}{1} & Expanding & $\begin{array}{c}\mathrm{M} \\
\mathrm{A}\end{array}$ & & $\begin{array}{l}2.18 \\
2.48\end{array}$ & $\begin{array}{l}2.60 \\
3.14\end{array}$ & $\begin{array}{l}2.44 \\
2.74\end{array}$ & $\begin{array}{l}3.20^{* *} \\
3.27\end{array}$ \\
\hline & Mature & $\begin{array}{l}\mathrm{M} \\
\mathrm{A}\end{array}$ & & $\begin{array}{l}1.33 \\
1.20\end{array}$ & $\begin{array}{l}1.54 \\
2.10\end{array}$ & $\begin{array}{l}0.87 \\
1.23\end{array}$ & $\begin{array}{l}1.57 \\
1.85\end{array}$ \\
\hline \multirow[t]{2}{*}{2} & Expanding & $\begin{array}{l}\mathrm{M} \\
\mathrm{A}\end{array}$ & & $\begin{array}{l}2.43 \\
3.40\end{array}$ & $\begin{array}{l}3.20 \\
3.80\end{array}$ & $\begin{array}{l}2.98 \\
3.07\end{array}$ & $\begin{array}{l}4.03^{* *} \\
4.36\end{array}$ \\
\hline & Mature & $\begin{array}{c}\mathrm{M} \\
\mathrm{A}\end{array}$ & & $\begin{array}{l}1.07 \\
1.34\end{array}$ & $\begin{array}{l}1.57 \\
1.70\end{array}$ & $\begin{array}{l}1.59 \\
1.62\end{array}$ & $\begin{array}{l}1.98^{*} \\
2.31\end{array}$ \\
\hline \multirow[t]{2}{*}{3} & Expanding & $\begin{array}{l}\mathrm{M} \\
\mathrm{A}\end{array}$ & & $\begin{array}{l}3.19 \\
2.42\end{array}$ & $\begin{array}{l}2.98 \\
2.90\end{array}$ & $\begin{array}{l}3.40 \\
2.50\end{array}$ & $\begin{array}{l}4.28^{*} \\
3.89\end{array}$ \\
\hline & Mature & $\begin{array}{c}\mathrm{M} \\
\mathrm{A}\end{array}$ & & $\begin{array}{l}1.53 \\
0.66\end{array}$ & $\begin{array}{l}1.69 \\
1.07\end{array}$ & $\begin{array}{l}1.87 \\
1.36\end{array}$ & $\begin{array}{l}2.65^{* *} \\
1.54^{*}\end{array}$ \\
\hline \multirow[t]{2}{*}{4} & Expanding & $\begin{array}{l}\mathrm{M} \\
\mathrm{A}\end{array}$ & & $\begin{array}{l}2.66 \\
3.06\end{array}$ & $\begin{array}{l}1.90 \\
3.40\end{array}$ & $\begin{array}{l}2.45 \\
2.73\end{array}$ & $\begin{array}{l}3.64 \\
3.66\end{array}$ \\
\hline & Mature & $\begin{array}{c}\mathrm{M} \\
\mathrm{A}\end{array}$ & & $\begin{array}{l}0.94 \\
1.53\end{array}$ & $\begin{array}{l}1.20 \\
0.99\end{array}$ & $\begin{array}{l}1.04 \\
1.98\end{array}$ & $\begin{array}{l}1.49 \\
1.99\end{array}$ \\
\hline \multirow[t]{2}{*}{5} & Expanding & $\begin{array}{c}\mathrm{M} \\
\mathrm{A}\end{array}$ & & $\begin{array}{l}2.91 \\
3.88\end{array}$ & $\begin{array}{l}3.54 \\
4.32\end{array}$ & $\begin{array}{l}2.76 \\
3.30\end{array}$ & $\begin{array}{l}3.07 \\
3.01\end{array}$ \\
\hline & Mature & $\begin{array}{l}\mathrm{M} \\
\mathrm{A}\end{array}$ & & $\begin{array}{l}1.14 \\
1.43\end{array}$ & $\begin{array}{l}1.71 \\
1.72\end{array}$ & $\begin{array}{l}2.07 \\
3.19^{*}\end{array}$ & $\begin{array}{l}3.25^{* *} \\
3.04\end{array}$ \\
\hline
\end{tabular}

${ }^{2} \mathrm{~A}$ : afternoon: $\mathrm{M}$ : morning.

*** Significantly different from the control in the same row at $P \leq$ 0.05 or 0.01 , respectively.

zero dose. Fourteen runs were used to calculate precision and sensitivity of the reducing sugar assay. The within- and between-assay cv were $8.7 \%$ and $19 \%$, respectively. The sensitivity of the assays was $5.0 \mathrm{meq}$ glucose.

\section{Results}

Leaf injury. On day 4, injury was visible on those plants treated with the combination of $\mathrm{SO}_{2}+\mathrm{NO}_{2}$. The injury was observed as a glazing of the abaxial surface of the middle and upper (which included the expanding leaflets) canopy leaflets. Visible injury consisting of interveinal abaxial necrotic areas was present. on day 10 in the middle canopy leaflets of the plants treated with $\mathrm{SO}_{2}$. Visible injury was absent on plants treated with $\mathrm{NO}_{2}$ alone.

Growth. Over the 10 days of exposure, significant differences in leaf, stem, and root growth were observed only between the control plants and those treated with $\mathrm{SO}_{2}+\mathrm{NO}_{2}$. Leaf area and leaf, stem, and root dry weights of the control plants followed significant quadratic growth patterns over time, while linear responses were obtained for the mixture of gases (Table 1). $\mathrm{SO}_{2}+\mathrm{NO}_{2}$ reduced leaf area and leaf dry weight. Leaf area was significantly reduced by day 2 , before significant decreases were observed in any of the other growth measurements. By day 6, leaf and stem dry weights were significantly reduced by the $\mathrm{SO}_{2}+\mathrm{NO}_{2}$ exposure. There was a significant reduction in root dry weight on day 4 of the exposure. The decrease in root dry weight was accompanied by a significant increase in leaf : root dry weight compared with control plants.
Net carbon dioxide exchange rates. Neither $\mathrm{SO}_{2}$ nor the mixture had an effect on net $\mathrm{CO}_{2}$ exchange rates at $0300 \mathrm{HR}$ (dark respiration period) (Table 2 ). However, dark respiration increased significantly in the $\mathrm{NO}_{2}$-treated plants, but only on day 10 .

The net $\mathrm{CO}_{2}$ exchange rate of the plants in $\mathrm{SO}_{2}+\mathrm{NO}_{2}$ decreased significantly compared to the control at $1100 \mathrm{HR}$ on days 5 and 8 (Table 2 ). A significant reduction in net $\mathrm{CO}_{2}$ exchange rate at $1900 \mathrm{HR}$ was observed on days 8 and 9 of plants in $\mathrm{SO}_{2}+\mathrm{NO}_{2}$ and on days $\mathrm{O}$ and 8 in $\mathrm{SO}_{2}$ alone (Table 2).

Water loss rates. Significant differences in net water loss rates were observed in the $\mathrm{NO}_{2}$ and the $\mathrm{SO}_{2}+\mathrm{NO}_{2}$ treatments (Table 3). The $\mathrm{NO}_{2}$-fumigated plants had a significantly higher water loss rate than the control plants during the 0300 HR measurement on day 9. No significant effects of $\mathrm{NO}_{2}$ were measured at $1100 \mathrm{HR}$ or $1900 \mathrm{HR}$. Plants exposed to $\mathrm{SO}_{2}+\mathrm{NO}_{2}$ began to lose water significantly faster than the control plants by 1900 HR on day 3. Increased net water loss rates continued until day 8 , although they were not always significantly different from the control.

Stomatal conductance. No significant differences in stomatal conductance between plants treated with pollutants and control plants were detected during the experiment (data not presented).

Leaf water potential. During the exposure period, significant responses were observed in the $\Psi_{\text {leaf }}$ of the $\mathrm{SO}_{2}+\mathrm{NO}_{2}$-treated plants (Table 4). No significant differences from control plants were detected for the $\mathrm{SO}_{2}$ or $\mathrm{NO}_{2}$ single gas treatments. About $6 \mathrm{~h}$ after beginning the exposure to the combination of $\mathrm{SO}_{2}+$ $\mathrm{NO}_{2}$ (afternoon of day 0 ), $\mathrm{P}$ was significantly reduced in the mature leaflets (Table 4), but this difference from the control did not continue.

$\psi_{\text {leaf }}$ of the mature leaflets in the mixture treatment was first observed to be significantly less than that of the control on the afternoon of day 1 (Table 4). This significant reduction continued, except for day 2 , in the afternoon and was noted for morning periods on days 8 and 10. The expanding leaflets also had a significant reduction in $\psi_{\text {leaf }}$ beginning on day 2 except for the morning of day 8 .

In the mature and expanding leaflets of the mixture-treated plants, $\pi$ was first observed to be significantly lower than that of the controls on the morning of day 1 (Table 4), and the difference was more consistent in mature than in expanding leaflets. This difference was observed on most of the measurement days in the morning and afternoon.

Reducing sugars. The concentration of reducing sugars in the expanding leaflets during the afternoons in any of the pollutant treatments did not differ significantly from the control leaflets (Table 5). Significantly higher reducing sugar concentrations in the mornings of days 2,3 , and 5 were observed in the mature leaflets exposed to $\mathrm{SO}_{2}+\mathrm{NO}_{2}$. During the morning observation, significant increases in reducing sugars over the control were observed only in the leaflets exposed to the combination of $\mathrm{SO}_{2}+\mathrm{NO}_{2}$. Increases in reducing sugar concentration were measured in the expanding leaflets on days 1, 2, and 3 and in the mature leaflets on days 3 and 5 (Table 5). In general, there was an increase in the concentration of reducing sugars in the leaflets that had been exposed to $\mathrm{SO}_{2}+\mathrm{NO}_{2}$ relative to control plants throughout the first half of the exposure.

\section{Discussion}

Sequential harvests of plants during an exposure to $\mathrm{SO}_{2}$ and/ or $\mathrm{NO}_{2}$ air pollution for 10 days permitted the identification of 
a series of events occurring in the plants. On day 1 , leaf $\pi$ was significantly lower in the plants treated with $\mathrm{SO}_{2}+\mathrm{NO}_{2}$ than in the control plants. This reduction was accompanied by a significant increase in the concentration of reducing sugars on a leaf-area basis in the expanding leaflets. Sulphur dioxide alone at low concentrations $\left(<3.06 \mu \mathrm{l} \cdot\right.$ liter $\left.^{-1}\right)$ has been shown to cause an increase in free sugar levels and starch levels in Phaseolus vulgaris L. (Koziol and Jordan, 1978).

A significant decrease in $\psi_{\text {leaf }}$ was not evident until day 2 when leaf area also was significantly reduced. Potato plants undergoing drought stress also exhibit decreases in leaf area growth (Gandar and Tanner, 1976). Leaf expansion of 'Russet Burbank' plants grown under greenhouse conditions was reduced at a $\psi_{\text {leaf }}$ of about $-300 \mathrm{kPa}$, with the cessation of expansion occurring at approximately - $500 \mathrm{kpa}$. Hsiao (1973) noted that cell expansion is one of the plant processes most sensitive to drought stress. McCree et al. (1984) showed that leaf expansion in water-stressed sorghum plants was not closely related to pressure (turgor) potential in the exposed lamina. Cell enlargement may not always be closely correlated with turgor because it depends on metabolic processes affecting cell wall extensibility and a supply of solutes, as well as on the physical processes affecting the water supply and cell turgor (Kramer, 1988). In our study, it is unlikely that $P$ had much of a role in allowing for the reduction in leaf area, because $\mathrm{P}$ did not decrease significantly throughout much of the exposure period. The reduction in leaf expansion rate may have been the result of pollutant by-products affecting cell wall synthesis or the lack of available organic substances to maintain growth (Munns and Weir, 1981). As a consequence of decreased leaf area, leaf area index (leaf area per unit land area) will be reduced, limiting the crop's assimilation of $\mathrm{CO}_{2}$ and ultimately affecting dry-matter yield of the tuber (Scott and Wilcockson 1978).

Some species have an endogenous ability to control intracellular solute contents by increasing the number of osmotically active particles as water becomes less available to the cell (a decrease in total $\left.\psi_{\text {leaf }}\right)$ (Shackel and Hall, 1983). Osmotic adjustment is the term used to describe this action (Reed, 1984), and it usually occurs under slowly developing drought stress conditions (Kramer, 1983). As $\pi$ decreases (becomes more negative), an inwardly directed force is established that creates a tendency for water to enter the cell (Reed, 1984) and allows for the maintenance of turgor and turgor-dependent processes, i.e., cell expansion, stomatal opening, and photosynthesis (Turner and Jones, 1980) at a significantly lower water potential (Kramer, 1983). Whether osmotic adjustment was occurring in the pollutant-stressed potato leaflets is uncertain. Three characteristics of the process were evident: turgor was maintained, $\pi$ decreased, and the concentration of reducing sugars (solutes) increased. More research must be performed on potato plants to determine if the reducing sugars are a direct result of photosynthesis or the products of starch degradation. Vos and Groenwold (1988) have reported in 'Bintje' potato that leaves appearing during a drought stress period have higher $\pi$ values at full turgidity than those leaves already present during the stress period. The effects of $\mathrm{SO}_{2}+\mathrm{NO}_{2}$ and drought on the potato plant appear to be similar.

A significant decline in photosynthesis rate was measured by day 5 of the $\mathrm{SO}_{2}+\mathrm{NO}_{2}$ exposure. Boyer (1970) demonstrated in soybean and Hsiao (1973) stated that, under drought stress, leaf enlargement is often reduced or stopped before photosynthesis is greatly reduced. Leaf area was significantly reduced by day 2 in the potato plants. By day 3 there was a significant increase in rate of water loss. Since stomatal conductance during the exposure did not change significantly in the treatments with pollutants compared with the control, and the rate of water loss rose, the stomates probably had very little or no influence on the reduction in the rate of photosynthesis. Another possible cause for the decrease in net $\mathrm{CO}_{2}$ exchange rate may have been an increase in respiration rate (Reinert, 1984). Since no significant mixture-induced increases were observed in the dark respiration rates, they were probably not the cause for the reduction in the photosynthesis rates. Thus, reduction of photosynthesis could have been caused by direct inhibition at the metabolic level (Amundson and MacLean, 1982; Carlson, 1983).

A decrease in net $\mathrm{CO}_{2}$ exchange rate was observed not only in the plants exposed to the mixture but also, and with greater magnitude, in the $\mathrm{SO}_{2}$-treated plants. The injurious effects of $\mathrm{SO}_{2}$ may have been the major contributor to the measured reduction in photosynthesis rates of the plants treated with $\mathrm{SO}_{2}$ $+\mathrm{NO}_{2}$.

The significant increase in water loss rate of the plants under $\mathrm{SO}_{2}+\mathrm{NO}_{2}$ was observed without a detectable increase in stomatal conductance. This result has also been observed in Betula sp. by Neighbour et al. (1988) who stated that the increase in water loss rate probably was not due to abnormal functioning of the stomata but may have been due to areas of gaping stomata or to cuticular injury. Pande and Oates (1986) found that after 4 days of exposure to $0.10 \mu \mathrm{SO}_{2}+\mathrm{NO}_{2}$ /liter, injury in Commelina communis L. occurred abaxially in the form of cuticular erosion probably accompanied by an increase in cuticular transpiration. By day 4 in our experiment, leaf injury was observed as abaxial leaf glazing. Since only the abaxial leaf surface was measured for stomatal resistance, it may have reflected injury to the adaxial cuticular surface and may have accounted for the increase in water loss rate. Further studies should consider observing stomatal resistances on both leaf surfaces. In addition, histological studies should be conducted to determine the extent of leaf injury.

By day 4, leaf injury was visible and root fresh and dry weights were significantly reduced by the $\mathrm{SO}_{2}+\mathrm{NO}_{2}$ treatment. The combination of $0.11 \mu \mathrm{SO}_{2}+0.11 \mu \mathrm{NO} \mathrm{NO}_{2} /$ liter for 2 weeks on tomato also caused a significant decrease in root growth without leaf fresh and dry weights diminishing at the same rate (Marie and Ormrod, 1984). Whitmore and Mansfield (1983) also observed that the combination of $\mathrm{SO}_{2}+\mathrm{NO}_{2} \mathrm{de}$ creased the partitioning of dry matter to the roots in Poa pratensis L. 'Monopoly'. Gould and Mansfield (1988) demonstrated in winter wheat that $\mathrm{SO}_{2}+\mathrm{NO}_{2}$ caused greater reductions in root dry weight than in shoot dry weight. Less ${ }^{14} \mathrm{C}$ assimilate was received by the roots than the shoots, suggesting an inhibition of translocation either in the stem or in the source leaves. Significant increases in leaf : hypocotyl dry weight ratios were observed in six radish cultivars exposed to $0.15 \mu \mathrm{l} \mathrm{NO}_{2} /$ liter, indicating a significant alteration of assimilate partitioning between source and sink areas of the plant (Godzik et al., 1985).

Tingey (1978) demonstrated that $\mathrm{O}_{3}$ altered root growth and root processes by first reducing photosynthesis and changing metabolic pathways. In our experiment with $\mathrm{SO}_{2}+\mathrm{NO}_{2}$, no significant reductions in photosynthesis rate were observed before reduced root growth. Tingey (1978) stated that reductions in root growth eventually should be expressed as reduced shoot growth. We observed significant reductions in shoot fresh and dry weights after reduced root growth was recorded.

The effects of $\mathrm{SO}_{2}+\mathrm{NO}_{2}$ in combination on potato plants involved a sequence of two types of injury: hidden and vis- 
ible. Heath (1980) defined hidden injury as biochemical or physiological alterations resulting in lowered plant productivity without visible injury. Malhotra and Khan (1984) state that the progression of hidden or invisible injury begins at the biochemical level (interference with photosynthesis, respiration, lipid and protein synthesis, etc.), subsequently progresses to the ultrastructural level (disorganization of cellular membranes), and then to the cellular level (cell-wall, mesophyll, and nuclear breakdown). In our experiment, hidden injury began with the decrease in $\pi$, which was at least partially associated with an increase in reducing-sugar concentration. This change was followed by reduced leaf area and then decreased net $\mathrm{CO}_{2}$ exchange. Reductions in root fresh and dry weights were evident after $\approx 4$ days of exposure, slightly before or about the same time as injury became visible. Under normal circumstances, the increased reducing sugars that were found in the leaves of treated plants, would be translocated to the roots and used for growth. However, sugars retained by the leaves were probably not available for growth even by the leaves (Munns and Weir, 1981).

Combinations of $\mathrm{SO}_{2}$ and $\mathrm{NO}_{2}$ occur in the natural environment (Ashenden, 1979a; Tingey et al., 1971). Our results demonstrate that their interaction can cause reductions in growth and dry-matter partitioning not observed with one gas. In the potato, the osmotic component of water potential appears to be one of the first factors detrimentally involved. The causes of build-up of reducing sugar and its relationship to water potential and growth effects should be an objective for further research to better understand the mechanism of joint 'action of $\mathrm{SO}_{2}$ and $\mathrm{NO}_{2}$ in plants.

\section{Literature Cited}

Amundson, R.G. and D.C. MacLean. 1982. Influence of oxides of nitrogen on crop growth and yield: An overview, p. 501-510. In: T. Schneider and L. Grant (eds.). Air pollution by nitrogen oxides. Elsevier Scientific, Amsterdam.

Amundson, R.G. and L.H. Weinstein. 1981. Joint action of sulphur dioxide and nitrogen dioxide on foliar injury and stomatal behaviour in soybean. J. Environ. Qual. 10:204-206.

Ashenden, T.W. 1979a. The effects of long-term exposures to $\mathrm{SO}_{2}$ and $\mathrm{NO}_{2}$ pollution on growth of Dactylis glomerata L. and Poa pratensis L. Environ. Polhrt. 18:249-258.

Ashenden, T.W. 1979b. Effects of $\mathrm{SO}_{2}$ and $\mathrm{NO}_{2}$ pollution on transpiration in Phaseolus vulgaris L. Environ. Pollut. 18:45-50.

Beringer, H., H.E. Haeder, and M. Lindhauer. 1983. Water relationships and incorporation of ${ }^{14} \mathrm{C}$ assimilates in tubers of potato plants differing in potassium nutrition. Plant Physiol. 73:956-960.

Black, V.J. and M.H. Unsworth. 1979. Effects of low concentrations of sulphur dioxide on gas exchange of plants and dark respiration of VIcia faba. J. Expt. Bet. 30:473-483.

Boyer, J.S. 1970. Leaf enlargement and metabolic rates in corn, soybean, and sunflower at various leaf water potentials. Plant Physiol. 46:233-235.

Carlson, R.W. 1983. Interaction between $\mathrm{SO}_{2}$ and $\mathrm{NO}_{2}$ and their effects on photosynthetic properties of soybean, Glycine max. Environ. Pollut. (Ser. A). 32:11-38.

Elkiey, T. and D.P. Ormrod. 1979. Ozone and sulphur dioxide effects on leaf water potential of Petunia. Z. Pflanzenphysiol. 91:177181 .

Fisheries and Environment Canada. 1976. Criteria for national air quality objectives. Sulphur dioxide, suspended particulate, carbon monoxide, oxidants (ozone) and nitrogen dioxide. Govt. Can., Ottawa.

Foster, K. W., H. Timm, C.K. Labanauskas, and R.J. Oshima. 1983. Effects of ozone and sulfur dioxide on tuber yield and quality of potatoes. J. Environ. Qual. 12:75-80.
Gandar, P.W. and C.B. Tanner. 1976. Leaf growth, tuber growth and water potential in potatoes. Crop Sci. 16:534-538.

Godzik, S., M.R. Ashmore, and J.N.B. Bell. 1985. Responses of radish cultivars to long-term and short-term exposures to sulphur dioxide, nitrogen dioxide, and their mixture. New Phytol. 100:191197.

Gould, R.P. and T.A. Mansfield. 1988. Effects of sulphur dioxide and nitrogen dioxide on growth and translocation in winter wheat. J. Expt. Bet. 39:389-399.

Harris, F.S. and D.W. Pittman. 1923. Irrigation experiments with potatoes. Utah Agr. Expt. Sta. Bul. 187.

Heath, R.L. 1980. Initial events in injury to plants by air pollutants. Annu. Rev. Plant Physiol. 31:395-431.

Hoagland, D.R. and D.I. Amen. 1950. The water-culture method for growing plants without soil. Calif. Agr. Expt. Sta. Circ. 347.

Hsiao, T.C. 1973. Plant responses to water stress. Annu. Rev. Plant Physiol. 24:519-570.

Koziol, M.J. and C.F. Jordan. 1978. Changes in carbohydrate levels in red kidney bean (Phaseolus vulgaris L.) exposed to sulphur dioxide. J. Expt. Bet. 29:1037-1043.

Kramer, P.J. 1983. Water relations of plants. Academic, Toronto.

Kramer, P.J. 1988. Changing concepts regarding plant water relations. Plant Cell \& Environ. 11:565-568.

Le Sueur-Brymer, N.M. 1982. Effects of ozone and sulphur dioxide singly or in combination on net photosynthesis, transpiration and growth of Glycine max (L.) Merr. MSC Thesis, Univ. of Guelph, Guelph, Ont.

Madore, M.A. 1984. Partitioning and transport of glycolate pathway carbon in $\mathrm{C}_{3}$ source leaves. PhD Diss., Univ. of Guelph, Guelph, Ont.

Malhotra, S.S. and A.A. Khan. 1984. Biochemical and physiological impact of major pollutants, p. 113-157. In: M. Treshow (cd.). Air pollution and plant life. Wiley, Toronto.

Marie, B.A. and D.P. Ormrod. 1984. Tomato growth with continuous exposure to sulphur dioxide and nitrogen dioxide. Environ. Pollut. (Ser. A) 33:257-265.

McCree, K. J., C.E. Kallsen, and S.G. Richardson. 1984. Carbon balance of sorghum plants during osmotic adjustment to water stress. Plant Physiol. 76:898-902.

Munns, R. and R. Weir. 1981. Contribution of sugars to osmotic adjustment in elongating and expanding zones of wheat leaves during moderate water deficits at two light levels. Austral. J. Plant Physiol. 8:93-105.

Neighbour, E.A., D.A. Cottam, and T.A. Mansfield. 1988. Effects of sulphur dioxide and nitrogen dioxide on the control of water loss by birch (Betula spp. ). New Phytol. 108:149-157.

Ormrod, D.P. 1982. Air pollutant interactions in mixtures, p. 307331. In: M.H. Unsworth and D.P. Ormrod (eds.). Effects of gaseous air pollution in agriculture and horticulture. Butterworths, Toronto.

Ormrod, D. P., D.T. Tingey, and M. Gumpertz. 1983. Covariate measurements for increasing the precision of plant response to $\mathrm{O}_{3}$ and $\mathrm{SO}_{2}$. HortScience 18:896-898.

Pande, P.C. and K. Oates. 1986. SEM analysis of Commelina communis L. leaves after exposure to $\mathrm{SO}_{2}$ and $\mathrm{NO}_{2}$ pollution. Environ. Pollut. (Ser. A) 42:353-360.

Pesez, M. and J. Bartos. 1974. Clinical and biochemical analysis. vol. 1. Calorimetric and fluorimetric analysis of organic compounds and drugs. Marcel Dekker, New York.

Petitte, J.M. 1986. The effects of sulphur dioxide and nitrogen dioxide on growth, physiology, and water status of the potato (Solanam tuberosum L.) plant. PhD Diss., Univ. of Guelph, Guelph, Ontario.

Petitte, J.M. and D.P. Ormrod. 1988. Effects of sulphur dioxide and nitrogen dioxide on shoot and root growth of Kennebec and Russet Burbank potato plants. Amer. Potato J. 65:517-527.

Prange, R.K. and D.P. Ormrod. 1983. Differential response in the water status of immature and mature fronds of the ostrich fern (Mat- 
teuccia struthiopteris (L) Todaro) to a mild water stress. Plant Physiol. 7296-98.

Reed, R.H. 1984. Use and abuse of osmo-terminology. Plant Cell \& Environ. 7:165-170.

Reinert, R.A. 1984. Plant response to air pollutant mixtures. Annu. Rev. Phytopath. 22421442.

Rodbard, D. 1971. Statistical aspects of radioimmunoassay, p. 204259. In: W.D. Odell and W.H. Daghaday (eds.). Principles of competitive protein binding assays. J.B. Lippineott, Philadelphia.

Scott, R.K. and S.J. Wilcockson. 1978. Application of physiological and agronomic principles to the development of the potato industry, p. 678-704. In: P.M. Harris (cd.). The potato crop. Chapman and Hall, London.

Shackel, K.A. and A.E. Hall. 1983. Comparison of water relations and osmotic adjustment in sorghum and cowpea under field conditions. Austral. J. Plant Physiol. 10:423-435.

Sinn, J.P. and E.J. Pen. 1984. Impact of repeated nitrogen dioxide exposures on composition and yield of potato foliage and tubers. J.

Amer. Soc. Hort. Sci. 109:481-484.
Snedecor, G.W. and W.G. Cochran. 1967. Statistical methods. Iowa State Univ. Press, Ames.

Tingey, D.T. 1978. Effects of ozone on root processes. Calif. Air Environ. 7:5

Tingey, D. T., R.A. Reinert, J.A. Dunning, and W.W. Heck. 1971. Vegetation injury from the interaction of nitrogen dioxide and sulfur dioxide. Phytopathology 61:1506-1511.

Turner, N.C. and M.M. Jones. 1980. Turgor maintenance by osmotic adjustment: A review and evaluation, p. 87-103. In: N.C. Turner and P.J. Kramer (eds.). Adaptation of plants to water and high temperature stress. Wiley, Toronto.

Vos, J. and J. Groenwold. 1988. Water relations of potato leaves. 1. Diurnal changes, gradients in the canopy, and effects of leaf-insertion number, cultivar and drought. Ann. Bet. 62:363-371.

Whitmore, M.E. and T.A. Mansfield. 1983. Effects of long-term exposures to $\mathrm{SO}_{2}$ and $\mathrm{NO}_{2}$ on Poa pratensis and other grasses. Environ. Pollut. (Ser. A) 32:217-235.

Wiebe, FLH. and H. Al-Saadi. 1976. Matric bound water of water tissue from succulents. Physiol. Plant. 36:47-51. 